\title{
Some new sharp bounds for the spectral radius of a nonnegative matrix and its application
}

\author{
Jun He*, Yan-Min Liu, Jun-Kang Tian and Xiang-Hu Liu
}

"Correspondence: hejunfan1@163.com School of Mathematics, Zunyi Normal College, Zunyi, Guizhou 563006, P.R. China

\begin{abstract}
In this paper, we give some new sharp upper and lower bounds for the spectral radius of a nonnegative irreducible matrix. Using these bounds, we obtain some new and improved bounds for the signless Laplacian spectral radius of a graph or a digraph.
\end{abstract}

MSC: 05C50; 05C35; 05C20; 15A18

Keywords: nonnegative matrix; graph; digraph; spectral radius

\section{Introduction}

Let $G=(V, E)$ be a graph with vertex set $V(G)=\left\{v_{1}, \ldots, v_{n}\right\}$ and edge set $E(G)$. Let $N=\{1, \ldots, n\}$, for $i \in N$. We assume that $d_{i}$ is the degree of vertex $v_{i}$. Let $D(G)=$ $\operatorname{diag}\left(d_{1}, d_{2}, \ldots, d_{n}\right)$ be the degree diagonal matrix of the graph $G$ and $A(G)=\left(a_{i j}\right)$ be the adjacency matrix of the graph $G$. Then the matrix $Q(G)=D(G)+A(G)$ is called the signless Laplacian matrix of the graph $G$. The largest modulus of eigenvalues of $Q(G)$ is denoted by $\rho(G)$, which is also called the signless Laplacian spectral radius of $G$.

Let $\vec{G}=(V, E)$ be a digraph with vertex set $V(\vec{G})=\left\{v_{1}, \ldots, v_{n}\right\}$ and $\operatorname{arc} \operatorname{set} E(\vec{G})$. Let $d_{i}^{+}$be the out-degree of vertex $v_{i}, D(\vec{G})=\operatorname{diag}\left(d_{1}^{+}, d_{2}^{+}, \ldots, d_{n}^{+}\right)$be the out-degree diagonal matrix of the digraph $\vec{G}$, and $A(\vec{G})=\left(a_{i j}\right)$ be the adjacency matrix of the digraph $\vec{G}$. Then the matrix $Q(\vec{G})=D(\vec{G})+A(\vec{G})$ is called the signless Laplacian matrix of the digraph $\vec{G}$. The largest modulus of eigenvalues of $Q(\vec{G})$ is denoted by $\rho(\vec{G})$, which is also called the signless Laplacian spectral radius of $\vec{G}$.

In recent decades, there are many bounds on the signless Laplacian spectral radius of a graph (digraph) [1-3]. Let $m_{i}=\frac{\sum_{i \sim} d_{j}}{d_{i}}$ be the average degree of the neighbours of $v_{i}$ in $G$ and $m_{i}^{+}=\frac{\sum_{i \sim j} d_{j}^{+}}{d_{i}^{+}}$be the average out-degree of the out-neighbours of $v_{i}$ in $\vec{G}$. In this paper, we assume that the graph (digraph) is simple and connected (strong connected).

In 2013, Maden, Das, and Cevik [4] obtained the following bounds for the signless Laplacian spectral radius of a graph:

$$
\rho(G) \leq \max _{i \sim j}\left\{\frac{d_{i}+2 d_{j}-1+\sqrt{\left(d_{i}-2 d_{j}+1\right)^{2}+4 d_{i}}}{2}\right\} .
$$

(0) The Author(s) 2017. This article is distributed under the terms of the Creative Commons Attribution 4.0 International License (http://creativecommons.org/licenses/by/4.0/), which permits unrestricted use, distribution, and reproduction in any medium, provided you give appropriate credit to the original author(s) and the source, provide a link to the Creative Commons license, and indicate if changes were made. 
In 2016, Xi and Wang [5] obtained the following bounds for the signless Laplacian spectral radius of a digraph:

$$
\rho(\vec{G}) \leq \max _{i \sim j}\left\{\frac{d_{i}^{+}+2 d_{j}^{+}-1+\sqrt{\left(d_{i}^{+}-2 d_{j}^{+}+1\right)^{2}+4 d_{i}^{+}}}{2}\right\} .
$$

In this paper, we improve the bounds for the signless Laplacian spectral radius of a graph (digraph) that are given in (1) and (2).

\section{Main result}

In this section, some upper and lower bounds for the spectral radius of a nonnegative irreducible matrix are given. We need the following lemma.

Lemma 2.1 ([6]) Let $A$ be a nonnegative matrix with the spectral radius $\rho(A)$ and the row sum $r_{1}, r_{2}, \ldots, r_{n}$. Then $\min _{1 \leq i \leq n} r_{i} \leq \rho(A) \leq \max _{1 \leq i \leq n} r_{i}$. Moreover, if the matrix $A$ is irreducible, then the equalities hold if and only if

$$
r_{1}=r_{2}=\cdots=r_{n}
$$

Theorem 2.1 Let $A=\left(a_{i j}\right)$ be an irreducible and nonnegative matrix with $a_{i i}=0$ for all $i \in N$ and the row sum $r_{1}, r_{2}, \ldots, r_{n}$. Let $B=A+M$, where $M=\operatorname{diag}\left(t_{1}, t_{2}, \ldots, t_{n}\right)$ with $t_{i} \geq 0$ for any $i \in N, s_{i}=\sum_{j=1}^{n} a_{i j} r_{j}, s_{i j}=s_{i}-a_{i j} r_{j}$. Let $\rho(B)$ be the spectral radius of $B$ and let

$$
f(i, j)=\frac{t_{i}+t_{j}+\frac{s_{i j}}{r_{i}}+\sqrt{\left(t_{i}-t_{j}+\frac{s_{i j}}{r_{i}}\right)^{2}+\frac{4 s_{j} a_{i j}}{r_{i}}}}{2},
$$

for any $i, j \in N$. Then

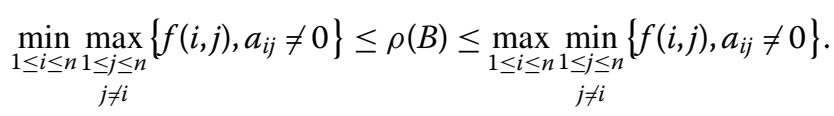

Moreover, either of the equalities in (3) holds if and only if $t_{i}+\frac{s_{i}}{r_{i}}=t_{j}+\frac{s_{j}}{r_{j}}$ for any distinct $i, j \in N$.

Proof Let $R=\operatorname{diag}\left(r_{1}, r_{2}, \ldots, r_{n}\right)$. Since the matrix $A$ is nonnegative irreducible, the matrix $R^{-1} B R$ is also nonnegative and irreducible. By the famous Perron-Frobenius theorem [6], there is a positive eigenvector $x=\left(x_{1}, x_{2}, \ldots, x_{n}\right)^{T}$ corresponding to the spectral radius of $R^{-1} B R$.

Upper bounds: Let $x_{p}>0$ be an arbitrary component of $x, x_{q}=\max \left\{x_{k}, 1 \leq k \leq n\right\}$. Obviously, $p \neq q, a_{p q} \neq 0$. By $R^{-1} B R x=\rho(B) x$, we have

$$
\rho(B) x_{p}=t_{p} x_{p}+\sum_{k=1, k \neq p}^{n} \frac{a_{p k} r_{k} x_{k}}{r_{p}} \leq t_{p} x_{p}+\frac{x_{q}}{r_{p}} \sum_{k=1}^{n} a_{p k} r_{k} \leq t_{p} x_{p}+\frac{x_{q} s_{p}}{r_{p}}
$$

Similarly, we have

$$
\rho(B) x_{q}=t_{q} x_{q}+\sum_{k=1, k \neq q}^{n} \frac{a_{q k} r_{k} x_{k}}{r_{q}} \leq\left(t_{q}+\frac{s_{q}-a_{q p} r_{p}}{r_{q}}\right) x_{q}+\frac{a_{q p} r_{p}}{r_{q}} x_{p} .
$$


By (4), (5), and $\rho(B)-t_{p}>0, \rho(B)-t_{q}>0$, we have

$$
\left(\rho(B)-t_{p}\right)\left(\rho(B)-t_{q}-\frac{s_{q}-a_{q p} r_{p}}{r_{q}}\right) \leq \frac{s_{p} a_{q p}}{r_{q}} .
$$

Therefore,

$$
\rho(B) \leq \frac{t_{p}+t_{q}+\frac{s_{q p}}{r_{q}}+\sqrt{\left(t_{p}-t_{q}-\frac{s_{q p}}{r_{q}}\right)^{2}+\frac{4 s_{p} a_{q p}}{r_{q}}}}{2} .
$$

This must be true for every $p \neq q$. Then

$$
\rho(B) \leq \min _{j \neq q} \frac{t_{j}+t_{q}+\frac{s_{q j}}{r_{q}}+\sqrt{\left(t_{j}-t_{q}-\frac{s_{q j}}{r_{q}}\right)^{2}+\frac{4 s_{j} a_{q j}}{r_{q}}}}{2} .
$$

This must be true for any $q \in N$. Then

$$
\rho(B) \leq \max _{1 \leq i \leq n} \min _{j \neq i}\left\{\frac{t_{i}+t_{j}+\frac{s_{i j}}{r_{i}}+\sqrt{\left(t_{i}-t_{j}+\frac{s_{i j}}{r_{i}}\right)^{2}+\frac{4 s_{j} a_{i j}}{r_{i}}}}{2}, a_{i j} \neq 0\right\} .
$$

Lower bounds: Let $x_{p}>0$ be an arbitrary component of $x, x_{q}=\min \left\{x_{k}, 1 \leq k \leq n\right\}$. Obviously, $p \neq q, a_{p q} \neq 0$. By $R^{-1} B R x=\rho(B) x$, we have

$$
\rho(B) x_{p}=t_{p} x_{p}+\sum_{k=1, k \neq p}^{n} \frac{a_{p k} r_{k} x_{k}}{r_{p}} \geq t_{p} x_{p}+\frac{x_{q}}{r_{p}} \sum_{k=1}^{n} a_{p k} r_{k} \geq t_{p} x_{p}+\frac{x_{q} s_{p}}{r_{p}} .
$$

Similarly, we have

$$
\rho(B) x_{q}=t_{q} x_{q}+\sum_{k=1, k \neq q}^{n} \frac{a_{q k} r_{k} x_{k}}{r_{q}} \geq\left(t_{q}+\frac{s_{q}-a_{q p} r_{p}}{r_{q}}\right) x_{q}+\frac{a_{q p} r_{p}}{r_{q}} x_{p} .
$$

By (9), (10), and $\rho(B)-t_{p}>0, \rho(B)-t_{q}>0$, we have

$$
\left(\rho(B)-t_{p}\right)\left(\rho(B)-t_{q}-\frac{s_{q}-a_{q p} r_{p}}{r_{q}}\right) \geq \frac{s_{p} a_{q p}}{r_{q}} .
$$

Therefore,

$$
\rho(B) \geq \frac{t_{p}+t_{q}+\frac{s_{q p}}{r_{q}}+\sqrt{\left(t_{p}-t_{q}-\frac{s_{q p}}{r_{q}}\right)^{2}+\frac{4 s_{p} a_{q p}}{r_{q}}}}{2} .
$$

This must be true for every $p \neq q$. Then

$$
\rho(B) \geq \max _{j \neq q} \frac{t_{j}+t_{q}+\frac{s_{q j}}{r_{q}}+\sqrt{\left(t_{j}-t_{q}-\frac{s_{q j}}{r_{q}}\right)^{2}+\frac{4 s_{j} a_{q j}}{r_{q}}}}{2} .
$$

This must be true for all $q \in N$. Then

$$
\rho(B) \geq \min _{1 \leq i \leq n} \max _{j \neq i}\left\{\frac{t_{i}+t_{j}+\frac{s_{i j}}{r_{i}}+\sqrt{\left(t_{i}-t_{j}+\frac{s_{i j}}{r_{i}}\right)^{2}+\frac{4 s_{j} a_{i j}}{r_{i}}}}{2}, a_{i j} \neq 0\right\} .
$$


From (4), (5), and $x_{p}>0$ as an arbitrary component of $x$, we get $x_{k}=x_{q}=x_{p}$ for all $k$. Then we see easily that the right equality holds in (8) for $t_{i}+\frac{s_{i}}{r_{i}}=t_{j}+\frac{s_{j}}{r_{j}}$ for any distinct $i, j \in N$. The proof of the left equality in (3) is similar to the proof of the right equality, and we omit it here.

Thus, we complete the proof.

\section{Signless Laplacian spectral radius of a graph}

In this section, we will apply Theorem 2.1 to obtain some new results on the signless Laplacian spectral radius $\rho(G)$ of a graph.

Theorem 3.1 Let $G=(V, E)$ be a simple connected graph on $n$ vertices. Then

$$
\begin{aligned}
& \min _{1 \leq i \leq n} \max _{i \sim j}\left\{\frac{d_{i}+2 d_{j}-1+\sqrt{\left(d_{i}-2 d_{j}+1\right)^{2}+4 d_{i}}}{2}\right\} \\
& \leq \rho(G) \leq \max _{1 \leq i \leq n} \min _{i \sim j}\left\{\frac{d_{i}+2 d_{j}-1+\sqrt{\left(d_{i}-2 d_{j}+1\right)^{2}+4 d_{i}}}{2}\right\} .
\end{aligned}
$$

Moreover, one of the equalities in (15) holds if and only if $G$ is a regular graph.

Proof We apply Theorem 2.1 to $Q(G)$. Let $t_{i}=0$ for any $i \in N$. Then $f(i, j)=$ $\frac{d_{i}+2 d_{j}-1+\sqrt{\left(d_{i}-2 d_{j}+1\right)^{2}+4 d_{i}}}{2}$. Thus (15) holds.

And the equality holds in (15) for regular graphs if and only if $G$ is a regular graph.

Remark 3.1 Obviously, we have

$$
\begin{array}{r}
\max _{1 \leq i \leq n} \min _{i \sim j}\left\{\frac{d_{i}+2 d_{j}-1+\sqrt{\left(d_{i}-2 d_{j}+1\right)^{2}+4 d_{i}}}{2}\right\} \\
\quad \leq \max _{i \sim j}\left\{\frac{d_{i}+2 d_{j}-1+\sqrt{\left(d_{i}-2 d_{j}+1\right)^{2}+4 d_{i}}}{2}\right\} .
\end{array}
$$

That is to say, our upper bound in Theorem 3.1 is always better than the upper bound (1) in [4].

Theorem 3.2 Let $G=(V, E)$ be a simple connected graph on $n$ vertices. Then

$$
\rho(G) \geq \min _{1 \leq i \leq n} \max _{i \sim j}\left\{\frac{d_{i}+d_{j}+m_{j}-d_{i} / d_{j}+\sqrt{\left(d_{i}-d_{j}-m_{j}+d_{i} / d_{j}\right)+4 d_{i}}}{2}\right\}
$$

and

$$
\rho(G) \leq \max _{1 \leq i \leq n} \min _{i \sim j}\left\{\frac{d_{i}+d_{j}+m_{j}-d_{i} / d_{j}+\sqrt{\left(d_{i}-d_{j}-m_{j}+d_{i} / d_{j}\right)+4 d_{i}}}{2}\right\} .
$$

Moreover, one of the equalities in (16), (17) holds if and only if $G$ is a regular graph or a bipartite semi-regular graph.

Proof We apply Theorem 2.1 to $Q(G)$. Let $t_{i}=d_{i}, s_{i}=\sum_{j=1}^{n} a_{i j} r_{j}=d_{i} m_{i}$ for any $1 \leq i \leq n$. Then $f(i, j)=\frac{d_{i}+d_{j}+m_{j}-d_{i} / d_{j}+\sqrt{\left(d_{i}-d_{j}-m_{j}+d_{i} / d_{j}\right)+4 d_{i}}}{2}$. Thus (16), (17) hold. 
And the equality holds if and only if $G$ is a regular graph or a bipartite semi-regular graph.

\section{Signless Laplacian spectral radius of a digraph}

In this section, we will apply Theorem 2.1 to obtain some new results on the signless Laplacian spectral radius $\rho(\vec{G})$ of a digraph.

Theorem 4.1 Let $\vec{G}=(V, E)$ be a strong connected digraph on $n$ vertices. Then

$$
\begin{aligned}
& \min _{1 \leq i \leq n} \max _{i \sim j}\left\{\frac{d_{i}^{+}+2 d_{j}^{+}-1+\sqrt{\left(d_{i}^{+}-2 d_{j}^{+}+1\right)^{2}+4 d_{i}^{+}}}{2}\right\} \\
& \leq \rho(\vec{G}) \leq \max _{1 \leq i \leq n} \min _{i \sim j}\left\{\frac{d_{i}^{+}+2 d_{j}^{+}-1+\sqrt{\left(d_{i}^{+}-2 d_{j}^{+}+1\right)^{2}+4 d_{i}^{+}}}{2}\right\} .
\end{aligned}
$$

Moreover, one of the equalities in (18) holds if and only if $\vec{G}$ is a regular digraph.

Proof We apply Theorem 2.1 to $Q(\vec{G})$. Let $t_{i}=0$ for any $1 \leq i \leq n$. Then $f(i, j)=$ $\frac{d_{i}^{+}+2 d_{j}^{+}-1+\sqrt{\left(d_{i}^{+}-2 d_{j}^{+}+1\right)^{2}+4 d_{i}^{+}}}{2}$. Then the inequality (18) holds.

And the equality holds in (18) if and only if $\vec{G}$ is a regular digraph.

Remark 4.1 Obviously, we have

$$
\begin{gathered}
\max _{1 \leq i \leq n} \min _{i \sim j}\left\{\frac{d_{i}^{+}+2 d_{j}^{+}-1+\sqrt{\left(d_{i}^{+}-2 d_{j}^{+}+1\right)^{2}+4 d_{i}^{+}}}{2}\right\} \\
\leq \max _{i \sim j}\left\{\frac{d_{i}^{+}+2 d_{j}^{+}-1+\sqrt{\left(d_{i}^{+}-2 d_{j}^{+}+1\right)^{2}+4 d_{i}^{+}}}{2}\right\} .
\end{gathered}
$$

That is to say, our upper bound in Theorem 4.1 is always better than the upper bound (2) in [5].

Theorem 4.2 Let $\vec{G}=(V, E)$ be a strong connected digraph on $n$ vertices. Then

$$
\rho(\vec{G}) \geq \min _{1 \leq i \leq n} \max _{i \sim j}\left\{\frac{d_{i}^{+}+d_{j}^{+}+m_{j}^{+}-d_{i}^{+} / d_{j}^{+}+\sqrt{\left(d_{i}^{+}-d_{j}^{+}-m_{j}^{+}+d_{i}^{+} / d_{j}^{+}\right)+4 d_{i}^{+}}}{2}\right\}
$$

and

$$
\rho(\vec{G}) \leq \max _{1 \leq i \leq n} \min _{i \sim j}\left\{\frac{d_{i}^{+}+d_{j}^{+}+m_{j}^{+}-d_{i}^{+} / d_{j}^{+}+\sqrt{\left(d_{i}^{+}-d_{j}^{+}-m_{j}^{+}+d_{i}^{+} / d_{j}^{+}\right)+4 d_{i}^{+}}}{2}\right\} .
$$

Moreover, one of the equalities in (19), (20) holds if and only if $\vec{G}$ is a regular digraph or a bipartite semi-regular digraph. 
Proof We apply Theorem 2.1 to $Q(\vec{G})$. Let $t_{i}=d_{i}^{+}, s_{i}=\sum_{j=1}^{n} a_{i j} r_{j}=d_{i}^{+} m_{i}^{+}$for any $1 \leq i \leq n$. Then $f(i, j)=\frac{d_{i}^{+}+d_{j}^{+}+m_{j}^{+}-d_{i}^{+} / d_{j}^{+}+\sqrt{\left(d_{i}^{+}-d_{j}^{+}-m_{j}^{+}+d_{i}^{+} / d_{j}^{+}\right)+4 d_{i}^{+}}}{2}$. Thus (19), (20) hold.

One sees easily that the equality holds if and only if $\vec{G}$ is a regular digraph or a bipartite semi-regular digraph.

\section{Conclusion}

In this paper, we give some new sharp upper and lower bounds for the spectral radius of a nonnegative irreducible matrix. Using these bounds, we obtain some new and improved bounds for the signless Laplacian spectral radius of a graph or a digraph which are better than the bounds in $[4,5]$.

\section{Acknowledgements}

Jun He is supported by the Science and Technology Foundation of Guizhou Province (Qian ke he Ji Chu [2016]1161); Guizhou Province Natural Science Foundation in China (Qian Jiao He KY [2016]255); the Doctoral Scientific Research Foundation of Zunyi Normal College (BS[2015]09); High-level Innovative Talents of Guizhou Province (Zun Ke He Ren Cai[2017]8). Yan-Min Liu is supported by National Science Foundations of China (71461027); Science and Technology Talent Training Object of Guizhou Province outstanding youth (Qian ke he ren zi [2015]06); Guizhou Province Natural Science Foundation in China (Qian Jiao He KY [2014]295); 2013, 2014 and 2015 Zunyi 15851 Talents Elite Project funding; Zhunyi Innovative Talent Team (Zunyi KH(2015)38). Tian is supported by Guizhou Province Natural Science Foundation in China (Qian Jiao He KY [2015]451); Science and Technology Foundation of Guizhou Province (Qian ke he J zi [2015]2147). Xiang-Hu Liu is supported by the Guizhou Province Department of Education fund (KY[2015]391, [2016]046); Guizhou Province Department of Education Teaching Reform Project [2015]337; Guizhou Province Science and Technology fund (Qian Ke He Ji Chu[2016]1160).

\section{Competing interests}

The authors declare that they have no competing interests.

\section{Authors' contributions}

All authors contributed equally to this work. All authors read and approved the final manuscript.

\section{Publisher's Note}

Springer Nature remains neutral with regard to jurisdictional claims in published maps and institutional affiliations.

Received: 3 August 2017 Accepted: 10 October 2017 Published online: 19 October 2017

\section{References}

1. Aouchiche, M, Hansen, P: Two Laplacians for the distance matrix of a graph. Linear Algebra Appl. 493, 21-33 (2013)

2. Bozkurt, SB, Bozkurt, D: On the signless Laplacian spectral radius of digraphs. Ars Comb. 108, 193-200 (2013)

3. Cui, SY, Tian, GX, Guo, JJ: A sharp upper bound on the signless Laplacian spectral radius of graphs. Linear Algebra Appl. 439, 2442-2447 (2013)

4. Maden, AD, Das, KC, Cevik, AS: Sharp upper bounds on the spectral radius of the signless Laplacian matrix of a graph. Appl. Math. Comput. 219, 5025-5032 (2013)

5. Xi, W, Wang, L: Sharp upper bounds on the signless Laplacian spectral radius of strongly connected digraphs. Discuss. Math., Graph Theory 36, 977-988 (2016)

6. Horn, RA, Johnson, CR: Matrix Analysis. Cambridge University Press, Cambridge (1985)

\section{Submit your manuscript to a SpringerOpen ${ }^{\circ}$ journal and benefit from:}

- Convenient online submission

- Rigorous peer review

- Open access: articles freely available online

- High visibility within the field

- Retaining the copyright to your article 